\title{
Evaluation of Asymptomatic Bacteriuria and Urinary Tract Infection in Patients With Primary Sjögren's Syndrome
}

\author{
Zeynep ÇETIN ${ }^{1}$ (D) Rıdvan MERCAN ${ }^{2}$, Ömer KARAŞAHIN ${ }^{3}$ OD, Abdurrahman TUFAN ${ }^{4}$, \\ Mehmet Akif ÖZTÜRK ${ }^{4}$ (D)
}

${ }^{1}$ Department of Endocrinology and Metabolism, Amasya University Sabuncuoğlu Şerefeddin Training and Research Hospital, Amasya, Turkey

${ }^{2}$ Department of Internal Medicine, Division of Rheumatology, Namık Kemal University Medical Faculty Hospital, Tekirdăg, Turkey

${ }^{3}$ Department of Clinical Bacteriology and Infection Diseases, Erzurum District Training and Research Hospital, Erzurum, Turkey

${ }^{4}$ Department of Internal Medicine, Division of Rheumatology, Gazi University Medical Faculty Hospital, Ankara, Turkey

\begin{abstract}
Objectives: This study aims to determine the frequency and risk factors of bacteriuria and urinary tract infection (UTI) in patients with primary Sjögren's syndrome (SS) and their differences from healthy individuals and rheumatoid arthritis (RA) patients.

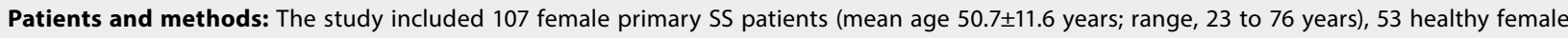
control subjects (mean age $46.8 \pm 15.5$ years; range 21 to 80 years), and 40 females with RA (mean age $51.7 \pm 14.2$ years; range, 25 to 79 years). Participants were questioned for UTI risk factors and symptoms. Middle stream urine samples were taken and cultured. All participants were examined with urinary symptom questioning survey of American Urological Association (AUA-7).

Results: The urine cultures were positive in 18 primary SS patients (16.8\%), eight RA patients (20\%), and two healthy controls (3.7\%). Escherichia coli, enterococci, Klebsiella, streptococci, and candida were detected in SS patients' cultures. Extended-spectrum beta-lactamase was positive in three cultures. Asymptomatic bacteriuria was not detected in any SS patient. The highest AUA-7 score was determined in SS group ( $p=0.031)$. Nineteen SS patients had vaginal dryness symptom and their AUA-7 scores were higher than the rest of the SS group. The risk of UTI development was not different between those who had or did not have vaginal dryness.

Conclusion: Urinary tract infections are seen more often in SS patients rather than normal population, which may be caused by SS' urinary system effects. It is difficult to distinguish between asymptomatic bacteriuria and infection because of the underlying urinary symptoms. Clinicians must be careful in patients receiving immunosuppressive therapy due to the high frequency of UTIs.

Keywords: Bacteriuria, infection, Sjögren's syndrome, urinary tract.
\end{abstract}

Sjögren's syndrome (SS) is an autoimmune disorder that may cause xerostomia, xerophthalmia, and vaginal xerosis because of lymphocytic infiltration of exocrine glands. ${ }^{1}$ It occurs as primary SS or secondary to another disease, raising glandular and extraglandular involvement. Most prominent glandular involvements are seen in eyes and oral mucosa, causing keratoconjunctivitis sicca and xerosis. Respiratory, digestive, and genitourinary systems are the other affected body parts; these may end up with dry noise, dry throat, esophagus atrophy, atrophic gastritis, subclinical pancreatitis, dyspareunia, and itching due to vaginal dryness. ${ }^{2}$

Urinary tract infection (UTI) is the invasion of pathogenic microorganisms into the urinary

Received: February 06, 2019 Accepted: December 24, 2019 Published online: June 25, 2020

Correspondence: Zeynep Çetin, MD. Amasya Üniversitesi Sabuncuoğlu Şerefeddin Eğitim ve Araştırma Hastanesi, Endokrinoloji ve Metabolizma Anabilim Dalı, 05200 Amasya, Türkiye. Tel: +90 536 - 7281262 e-mail: drzeynepcetin@gmail.com

Çetin Z, Mercan R, Karaşahin Ö, Tufan A, Öztürk MA. Evaluation of Asymptomatic Bacteriuria and Urinary Tract Infection in Patients With Primary Sjögren's Syndrome. Arch Rheumatol 2020;35(4):592-599. 
epithelium. UTI has high prevalence and frequent recurrence. Its therapy is difficult due to the development of antimicrobial resistance. The most common symptoms are dysuria, and frequent and urgent voiding wish. Immunosuppression is a risk factor for UTI like other infections. The gold standard method for the diagnosis is urine culture. The most frequent pathogen is Escherichia coli (E. coli) (85\%) followed by Staphylococcus saprophyticus (10\%). The critical point of treatment is choosing the antibiotic, which must be suitable for the pathogen detected in the antibiogram and have low risk for developing resistance. ${ }^{3}$

Asymptomatic bacteriuria is defined as the isolation of bacteria in significant amounts in the urine culture from a person without symptoms or signs of UTI. ${ }^{4}$ Isolation of the same bacterial strain $>10^{5}$ colony-forming unit $(\mathrm{cfu}) / \mathrm{mL}$ in two consecutive urine cultures in females is defined as asymptomatic bacteriuria. Isolation of $>10^{5} \mathrm{cfu} / \mathrm{mL}$ bacteria in the urine culture in males is sufficient for the diagnosis. In patients with internal catheters, this amount is lowered to $10^{2}$. There is no treatment indication with asymptomatic bacteriuria plus pyuria. ${ }^{5}$

Patients with systemic lupus erythematosus (SLE) and rheumatoid arthritis (RA) have been shown to have more urinary complaints. ${ }^{6,7}$ Patients with SS with RA have been shown to develop UTIs more often than patients with RA alone. ${ }^{8}$ To our knowledge, there are no studies investigating the incidence of UTI or asymptomatic bacteriuria in patients with primary SS in the literature. Therefore, in this study, we aimed to determine the frequency and risk factors of bacteriuria and UTI in patients with primary SS and their differences from healthy individuals and RA patients.

\section{PATIENTS AND METHODS}

This study was conducted at Gazi University Medicine Faculty Hospital between June 2011 and October 2012. The study included 107 female primary SS patients (mean age $50.7 \pm 11.6$ years; range, 23 to 76 years), 53 healthy female control subjects (mean age $46.8 \pm 15.5$ years; range 21 to 80 years), and 40 females with $\mathrm{RA}$ (mean age $51.7 \pm 14.2$ years; range, 25 to
79 years). Patients with SS, RA, and healthy subjects had normal renal functions, no risk factors for asymptomatic bacteriuria or UTI (such as pregnancy, immunosuppressive treatment, anatomic disorder, diabetes mellitus, pelvic relaxation, spinal cord injury, catheterization) or active infection in other systems during the study. The study protocol was approved by the Gazi University Medicine Faculty Hospital Ethics Committee. A written informed consent was obtained from each participant. The study was conducted in accordance with the principles of the Declaration of Helsinki.

The primary SS patients were diagnosed according to the criteria of the AmericanEuropean Consensus Group. ${ }^{9}$ American College of Rheumatology/European League Against Rheumatism 2010 diagnostic criteria were used for RA diagnosis. ${ }^{10}$ Healthy subjects were selected from the individuals who applied to the Internal Medicine Outpatient Clinic of Gazi University Hospital. They were over 18 years old, lived in Ankara, and did not have any systemic disorders or active infections.

Demographic characteristics, disease characteristics, and therapies were taken during face-to-face interviews and received from hospital records. Urinary tract infection symptoms and resistant UTI risk factors were questioned for each patient. The urinary system symptom questionnaire (AUA-7) of the American Urological Association was performed for all. AUA-7 symptom index includes seven questions covering frequency, nocturia, weak urinary stream, hesitancy, intermittence, incomplete emptying, and urgency; it helps understanding the severity of the symptoms. This questionnaire was validated for the Turkish language previously. ${ }^{11}$

Middle stream urine specimens were taken from the subjects after cleaning with external antiseptic and cultured. At the same time, routine urine analysis and urine microscopy (erythrocyte, leukocyte counts) were performed. Re-culture was taken in patients with bacterial growth in the first culture. Specimens were planted in the blood and eosin-methylene blue agar and incubated at $37^{\circ} \mathrm{C}$ for $18-24$ hours and then $>10^{5} \mathrm{cfu} / \mathrm{mL}$ of bacterial isolates were identified at the species level. Antibiotic 
susceptibility tests of isolated bacteria were performed according to the criteria of Clinical and Laboratory Standard Institute by KirbyBauer disc diffusion method.

\section{Statistical analysis}

Statistical analyses were performed using the SPSS for Windows version 16.0 (SPSS Inc., Chicago, IL, USA). Numerical variables were summarized with mean \pm standard deviation or median (minimum-maximum). Categorical variables were indicated by number and percentage. The Chi-square test or Fisher's exact test was used to determine whether there was any difference between the groups in terms of categorical variables. The Kolmogorov-Smirnov test was used to determine whether the normal variables showed normal distribution, and the homogeneity of the variance was examined by the Levene test. Differences between two independent groups in terms of numerical variables; in the case of parametric test assumptions, t-test was used for independent groups. In the absence of parametric test assumptions, Mann-Whitney $U$ test was used. The significance level was taken as $\mathrm{p}<0.05$.

\section{RESULTS}

All 203 participants (100\%) were females. There was no difference between the groups in terms of age $(p>0.05)$ (Table 1). Clinical characteristics of patients with primary SS were shown in Table 2. Steroid usage was less than $10 \mathrm{mg} /$ day (this dose is not immunosuppressive).

Urine culture was positive in 18 (16.8\%) of the 107 primary SS patients. All were confirmed by second culture. The pathogens were E. coli, enterococci, Klebsiella, streptococci, and candida. Extended-spectrum beta-lactamase (ESBL) positivity was detected in three cultures; two of them were E. coli and one was enterococcus. These 18 patients had UTI symptoms, while asymptomatic bacteriuria was not detected in any SS patient (Table 3).

Eight (20\%) of the RA cultures were found to have bacterial growth. All were confirmed by second culture as well. All eight patients had UTI symptoms. Asymptomatic bacteriuria was not detected (Table 4).

Escherichia coli was identified only in two $(3.7 \%)$ cultures of the healthy control group. Both

Table 1. American-European Consensus Group's international classification criteria for primary Sjögren's syndrome

I. Ocular symptoms: a positive response to at least one of the following questions:

1. Have you had daily, persistent, troublesome dry eyes for more than three months?

2. Do you have a recurrent sensation of sand or gravel in the eyes?

3. Do you use tear substitutes more than three times a day?

II. Oral symptoms: a positive response to at least one of the following questions:

1. Have you had a daily feeling of dry mouth for more than three months?

2. Have you had recurrently or persistently swollen salivary glands as an adult?

3. Do you frequently drink liquids to aid in swallowing dry food?

III. Ocular signs-that is, objective evidence of ocular involvement defined as a positive result for at least one of the following two tests:

1. Schirmer's I test, performed without anaesthesia ( $<5 \mathrm{~mm}$ in 5 minutes)

2. Rose Bengal score or other ocular dye score ( $>4$ according to van Bijsterveld's scoring system)

IV. Histopathology: In minor salivary glands (obtained through normal-appearing mucosa) focal lymphocytic sialoadenitis, evaluated by an expert histopathologist, with a focus score $>1$, defined as a number of lymphocytic foci (which are adjacent to normal-appearing mucous acini and contain more than 50 lymphocytes) per $4 \mathrm{~mm}^{2}$ of glandular tissue

$\mathrm{V}$. Salivary gland involvement: objective evidence of salivary gland involvement defined by a positive result for at least one of the following diagnostic tests:

1. Unstimulated whole salivary flow $(<1.5 \mathrm{~mL}$ in 15 minutes $)$

2. Parotid sialography showing the presence of diffuse sialectasis (punctate, cavitary or destructive pattern), without evidence of obstruction in the major ducts

3. Salivary scintigraphy showing delayed uptake, reduced concentration and/or delayed excretion of tracer

VI. Autoantibodies: presence in the serum of the following autoantibodies:

1. Antibodies to Ro(SSA) or La(SSB) antigens, or both

Ro(SSA): Sjögren's syndrome type A; La(SSB): Sjögren's syndrome type B. 
Table 2. American College of Rheumatology/European League Against Rheumatism 2010 classification criteria for rheumatoid arthritis

Target population (Who should be tested?): Patients

1) who have at least one joint with definite clinical synovitis (swelling)*

2) with the synovitis not better explained by another disease $\dagger$

Classification criteria for RA (score-based algorithm: add score of categories A-D; a score of $6 / 10$ is needed for classification of a patient as having definite RA) $\neq$

\section{A. Joint involvement§}

1 large joint 9

2-10 large joints

1-3 small joints (with or without involvement of large joints)\#

4-10 small joints (with or without involvement of large joints)

$>10$ joints (at least 1 small joint)***

B. Serology (at least one test result is needed for classification) $\dagger \dagger$ Negative RF and negative ACPA

Low-positive RF or low-positive ACPA

High-positive RF or high-positive ACPA

C. Acute-phase reactants (at least one test result is needed for classification)㸬

Normal CRP and normal ESR

D. Duration of symptoms§§ $<6$ weeks $>6$ weeks

RA: Rheumatoid arthritis; RF: Rheumatoid factor; ACPA: Anticitrullinated protein antibody; CRP: C-reactive protein; ESR: Erythrocyte sedimentation rate.

Criteria are aimed at classification of newly presenting patients. In addition, patients with erosive disease typical of RA with a history compatible with prior fulfillment of 2010 criteria should be classified as having RA. Patients with longstanding disease, including those whose disease is inactive (with or without treatment) who, based on retrospectively available data, have previously fulfilled 2010 criteria should be classified as having RA.

$\dagger$ Differential diagnoses vary among patients with different presentations, but may include conditions such as systemic lupus erythematosus, psoriatic arthritis, and gout. If it is unclear about relevant differential diagnoses to consider, an expert rheumatologist should be consulted.

\# Although patients with a score of $6 / 10$ are not classifiable as having RA, their status can be reassessed and criteria might be fulfilled cumulatively over time.

$\S$ Joint involvement refers to any swollen or tender joint on examination, which may be confirmed by imaging evidence of synovitis. Distal interphalangeal joints, first carpometacarpal joints, and first metatarsophalangeal joints are excluded from assessment. Categories of joint distribution are classified according to location and number of involved joints, with placement into highest category possible based on pattern of joint involvement.

पा Term "large joints" refers to shoulders, elbows, hips, knees, and ankles.

\# Term "small joints" refers to metacarpophalangeal joints, proximal interphalangeal joints, second through fifth metatarsophalangeal joints, thumb interphalangeal joints, and wrists.

** In this category, at least one of involved joints must be a small joint; other joints can include any combination of large and additional small joints, as well as other joints not specifically listed elsewhere (e.g., temporomandibular, acromioclavicular, sternoclavicular, etc.).

†† Negative refers to international unit (IU) values that are less than or equal to upper limit of normal (ULN) for laboratory and assay; low-positive refers to IU values that are higher than ULN but three times ULN for laboratory and assay; high-positive refers to IU values that are three times ULN for laboratory and assay. Where RF information is only available as positive or negative, a positive result should be scored as low positive for RF.

\# Normal/abnormal is determined by local laboratory standards.

$\S \S$ Duration of symptoms refers to patient self-report of duration of signs or symptoms of synovitis (e.g., pain, swelling, tenderness) of joints that are clinically involved at time of assessment, regardless of treatment status.

were negative for ESBL. These two patients were symptomatic as well. None of the 53 control subjects had asymptomatic bacteriuria.

Statistical analyses were performed among the three groups for age, presence of risk factors for UTI (diabetes mellitus, renal failure, immunosuppressive drug use, and urinary incontinence), average number of UTIs in last six months, leukocyturia, and number of positive cultures. There was no significant difference in terms of age, presence of risk factors, and leucocyturia. 
Table 3. Characteristics of study participants

\begin{tabular}{|c|c|c|c|c|c|c|c|c|c|c|c|c|}
\hline & \multicolumn{4}{|c|}{ Primary SS $(n=107)$} & \multicolumn{4}{|c|}{ Rheumatoid arthritis $(n=40)$} & \multicolumn{4}{|c|}{ Control $(n=53)$} \\
\hline & $\mathrm{n}$ & $\%$ & Mean \pm SD & Min-Max & $\mathrm{n}$ & $\%$ & Mean \pm SD & Min-Max & $\mathrm{n}$ & $\%$ & Mean \pm SD & Min-Max \\
\hline Age (year) & & & $50.7 \pm 11.6$ & $23-76$ & & & $51.7 \pm 14.2$ & $25-79$ & & & $46.8 \pm 15.5$ & $21-80$ \\
\hline \multicolumn{13}{|l|}{ Sex } \\
\hline Female & 107 & 100 & & & 40 & 100 & & & 53 & 100 & & \\
\hline \multicolumn{13}{|l|}{ Comorbidity } \\
\hline Hypertension & 35 & 32.7 & & & 10 & 25 & & & 17 & 32 & & \\
\hline Thyroid dysfunction & 22 & 22.5 & & & 0 & 0 & & & 11 & 20.7 & & \\
\hline Hyperlipidemia & 7 & 6.5 & & & 0 & 0 & & & 2 & 3.7 & & \\
\hline Coronary artery disease & 2 & 1.86 & & & 1 & 2.5 & & & 2 & 3.7 & & \\
\hline Asthma & 1 & 1.07 & & & 1 & 2.5 & & & 4 & 7.5 & & \\
\hline Autoimmune hepatitis & 2 & 1.86 & & & 0 & 0 & & & 0 & 0 & & \\
\hline Depression & 1 & 1.07 & & & 0 & 0 & & & 3 & 5.6 & & \\
\hline
\end{tabular}

Average number of UTIs in last six months was different between the groups. Binary comparisons revealed that this difference was due to SS $(p=0.013)$. This finding is important because it gives information about the susceptibility to infection in SS. Some patients with UTIs received

Table 4. Clinical and serologic findings of patients with primary Sjögren's syndrome at time of diagnosis

\begin{tabular}{|c|c|c|c|c|}
\hline & $\mathrm{n}$ & $\%$ & Mean \pm SD & Min-Max \\
\hline Symptom duration (year) & & & $5.6 \pm 3.8$ & $1-23$ \\
\hline Xerostomia & 72 & 67.3 & & \\
\hline Xerophthalmia & 81 & 75.7 & & \\
\hline Vaginal dryness & 19 & 17.8 & & \\
\hline Skin dryness & 31 & 29.0 & & \\
\hline Joint complaint & 100 & 93.5 & & \\
\hline Peripheral nervous system involvement & 10 & 9.3 & & \\
\hline Central nervous system involvement & 1 & 0.9 & & \\
\hline Pulmonary involvement & 2 & 1.86 & & \\
\hline Renal involvement & 2 & 1.86 & & \\
\hline Steroid usage & 12 & 11.2 & & \\
\hline Sedimentation $(\mathrm{mm} / \mathrm{h})$ & & & $36.9 \pm 1.9$ & $5-91$ \\
\hline C-reactive protein (mg/L) & & & $8.4 \pm 1.1$ & $0-59$ \\
\hline Rheumatoid factor positivity & 68 & 63.5 & & \\
\hline ANA positivity & 101 & 94.4 & & \\
\hline Anti-Ro (SSA) positivity & 68 & 63.5 & & \\
\hline Anti-Ro 52 positivity & 70 & 65.4 & & \\
\hline Anti-La (SSB) positivity & 41 & 38.3 & & \\
\hline \multicolumn{5}{|l|}{ Schirmer } \\
\hline Mild (10-15 mm/5 min) & 20 & 18.7 & & \\
\hline Moderate (5-10 mm/5 min) & 28 & 26.2 & & \\
\hline Advanced (<5 mm/5 min) & 29 & 27.1 & & \\
\hline \multicolumn{5}{|l|}{ Focus score } \\
\hline 0 & 11 & 10.3 & & \\
\hline 1 & 37 & 34.5 & & \\
\hline 2 & 32 & 29.9 & & \\
\hline 3 & 22 & 20.5 & & \\
\hline
\end{tabular}

SD: Standard deviation; Min: Minimum; Max: Maximum; ANA: Antinuclear antibody; Anti-Ro (SSA): AntiSjögren's syndrome type A; Anti-La (SSB): Anti-Sjögren's syndrome type B. 
Table 5. Comparison of the groups for age, risk factor, number of UTI within the last six months, leucocyturia and number of positive culture

\begin{tabular}{|c|c|c|c|c|c|c|c|c|c|c|c|c|c|}
\hline & \multicolumn{4}{|c|}{ Primary SS $(n=107)$} & \multicolumn{4}{|c|}{ Rheumatoid arthritis $(n=40)$} & \multicolumn{4}{|c|}{ Control $(n=53)$} & \multirow[b]{2}{*}{$p$} \\
\hline & $\mathrm{n}$ & $\%$ & Mean \pm SD & Min-Max & $\mathrm{n}$ & $\%$ & Mean \pm SD & Min-Max & $\mathrm{n}$ & $\%$ & Mean \pm SD & Min-Max & \\
\hline Age (year) & & & $50.7 \pm 11.6$ & & & & $51.7 \pm 14.2$ & $25-79$ & & & $46.8 \pm 15.5$ & $21-78$ & NS \\
\hline Risk factors & 17 & & & & 10 & & & & 7 & & & & NS \\
\hline $\begin{array}{l}\text { Average number of UTIs } \\
\text { in last six months }\end{array}$ & $27^{*}$ & & & & 4 & & & & 5 & & & & 0.013 \\
\hline Leukocyturia** & 21 & 19.6 & & & 14 & 35 & & & 17 & 32 & & & NS \\
\hline $\begin{array}{l}\text { Number of positive } \\
\text { culture }\end{array}$ & 18 & 16.8 & & & 8 & 20 & & & 2 & 3.7 & & & 0.04 \\
\hline
\end{tabular}

treatment while others did not. However, this data could not be provided.

The number of positive cultures was also different between the groups $(p=0.04)$. For this, binary comparisons were performed and the healthy control group was compared to the SS and RA groups separately. Both analyses showed $p=0.02$, indicating that the difference resulted from the SS and RA groups (Table 5).

In our study, AUA-7 test was used to question urinary system complaints. Kruskal-Wallis test was used to compare the three groups in terms of AUA-7 scores and $p$ was found to be 0.08; because of the differences in tendency, a binary analysis was performed with Mann-Whitney U test. The highest AUA-7 score was determined in the SS group (SS versus control, 4 versus 2, $\mathrm{p}=0.031$ ). Comparison of the control group with the RA group revealed $\mathrm{p}=0.073$ (RA versus control, 3 versus 2). No significant difference was found between SS and RA groups (SS versus RA, 4 versus $3, p=0.945$ ) (Table 6).

Mann-Whitney $U$ test was performed to investigate the relationship between vaginal dryness and AUA-7 scores in patients with

Table 6. Comparison of three groups of AUA-7 scores

\begin{tabular}{|c|c|c|c|c|}
\hline & SS ( $n=107)$ & $\mathrm{RA}(\mathrm{n}=40)$ & Control $(n=53)$ & \\
\hline & Median \pm IQR & Median \pm IQR & Median $\pm \mathrm{IQR}$ & $p$ \\
\hline AUA-7 & $4 \pm 6$ & $3 \pm 8$ & $2 \pm 4$ & 0.08 \\
\hline
\end{tabular}

primary SS. The AUA-7 score was higher in those with vaginal dryness, while the trend was not different $(p=0.057)$.

The Chi-square test to assess whether vaginal dryness was a risk factor for UTI showed no significant difference between those with and without dryness.

\section{DISCUSSION}

Infections are one of the main causes of morbidity and mortality in rheumatologic diseases. They are responsible for 30-50\% of clinical worsening in SLE. Patients are predisposed to underlying immunological abnormalities. Also, they are susceptible to infections due to the immunosuppressive treatment they receive. Infections affect the natural course of the primary disease and they may cause progression. Their clinical importance is increasing because they make it difficult to evaluate the treatment response. According to a retrospective study by Irlapati et al., ${ }^{12}$ infections were the most frequent cause of admission to the hospital for RA, SLE, and other rheumatic diseases. The most common infections were respiratory infections followed by UTIs.

The incidence of infections in SLE patients is quite high; they are the leading cause of death, while the first or second cause of hospitalization. ${ }^{13,14}$ UTIs are the most common infections in SLE. The most common cause is E. coli. ${ }^{15,16}$

Sjögren's syndrome is a chronic autoimmune disease with different clinical manifestations and 
its findings are related to both exocrine glands and other organ involvements. Renal disease due to interstitial involvement is well known in primary $\mathrm{SS}$ and it is related to the underlying autoimmune disease in secondary SS. ${ }^{17}$ The most common nephrological involvement in primary $\mathrm{SS}$ is interstitial nephritis, which is seen as type 1 renal tubular acidosis. ${ }^{12}$

The mucosal membranes' dryness in SS is associated with frequent infections. The risk of superficial infections in the dry eye is high because of the reduced lubricant and antibacterial activity of the teardrop. ${ }^{18}$

In both primary and secondary SS, studies on lower urinary tract infections are scarce. In a study conducted by Tishler et al., ${ }^{8}$ recurrent UTIs and pyuria were more frequent in patients with SS secondary to RA; about $80 \%$ of patients with vaginal involvement had an infection. The increased risk of infection was connected to defects in the urinary protective mechanisms, mucosal atrophy, and decreased urinary immunoglobulin A secretion.

In patients with SS, interstitial cystitis, which may be present in the form of frequent urination and suprapubic pain, is associated with lymphocytic infiltration of the bladder and can be conjectured as UTI; it is frequent and can be serious in SS patients. ${ }^{19}$ However, patients may also have real UTIs. In this study, we aimed to determine the differences in the frequency and risk factors of bacteriuria between healthy controls and RA and primary SS patients and to guide further studies to be conducted on this subject. These three groups were compared for age, risk factors for UTI, number of UTIs within the last six months, pyuria, number of positive cultures, and the detected pathogens. All the participants were questioned with AUA-7 questionnaire and whether vaginal dryness was a risk for UTI in patients with SS was investigated.

All the participants were chosen from females because UTI is mostly seen among females ${ }^{20}$ and our rheumatology policlinic's SS patients consist mostly of females. It has been determined that E. coli is the most common agent (it was only $E$. coli in the healthy control group). This result is compatible with the literature. ${ }^{21} \mathrm{ESBL}(+)$ growth was detected only in the SS group; however, it was interpreted that the SS group had more statistically significant UTIs in the last six months than the other groups, and that they were more likely to use antibiotics and thus become infected with resistant strains. ${ }^{22} \mathrm{RA}$ and $\mathrm{SS}$ groups had more positive cultures. This information is consistent with the literature in terms of RA and it forms new scientific data for SS. A comparison of the AUA-7 scores to assess urinary symptoms in the three groups showed that the SS patients had the highest score. Asymptomatic bacteriuria was not detected in any of the patients; however, urinary symptoms were evident, particularly in patients with primary SS. Thus, asymptomatic bacteriuria can be misinterpreted as UTI due to the urinary symptoms already present in SS patients. There is also a tendency to linear relationship between the AUA-7 score and vaginal dryness, and it is thought that a clear relationship can be shown by increasing the size of the sample.

Our study has some limitations. Firstly, we did not investigate male SS patients since our rheumatology clinic has more female patients. Secondly, the average number of infections gave an idea for susceptibility to UTI; however, not all patients had used antibiotics and we could not detect which antibiotics were used. Thirdly, the use of antibiotics may have influenced the results of the culture. Finally, the relationship between vaginal dryness and AUA-7 scores was found to be indifferent while the $\mathrm{p}$ value was 0.057 . This result may be due to the small number of the participants.

In conclusion, both cystitis symptoms are apparent in patients with primary SS and UTIs in these patients are more common compared to healthy controls, such as RA patients. Frequent UTI may be due to the urinary effects of the disease. Also, immunosuppressive treatment can increase UTI frequency in these patients. But, underlying urinary symptoms may make it difficult to distinguish between asymptomatic bacteriuria and UTI in these patients. Moreover, based on the linear relationship between the AUA-7 score and vaginal dryness, our study showed that primary SS predisposes to UTI. Further research is needed to investigate the impact of UTI on morbidity and mortality in patients with SS.

\section{Declaration of conflicting interests}

The authors declared no conflicts of interest with respect to the authorship and/or publication of this article. 


\section{Funding}

The authors received no financial support for the research and/or authorship of this article.

\section{REFERENCES}

1. Fox RI, Stern M, Michelson P. Update in Sjögren syndrome. Curr Opin Rheumatol 2000;12:391-8.

2. Moutsopoulos HM. Sjögren's Syndrome. New York: McGraw Hill Companies; 2012.

3. Dielubanza EJ, Schaeffer AJ. Urinary tract infections in women. Med Clin North Am 2011;95:27-41.

4. Rubin RH, Shapiro ED, Andriole VT, Davis RJ, Stamm WE. Evaluation of new anti-infective drugs for the treatment of urinary tract infection. Infectious Diseases Society of America and the Food and Drug Administration. Clin Infect Dis 1992;15:S216-27.

5. Nicolle LE, Bradley S, Colgan R, Rice JC, Schaeffer A, Hooton TM. Infectious Diseases Society of America guidelines for the diagnosis and treatment of asymptomatic bacteriuria in adults. Clin Infect Dis 2005;40:643-54.

6. Hidalgo-Tenorio C, Jiménez-Alonso J, de Dios Luna J, Tallada M, Martínez-Brocal A, Sabio JM. Urinary tract infections and lupus erythematosus. Ann Rheum Dis 2004;63:431-7.

7. Senior BW, Anderson GA, Morley KD, Kerr MA. Evidence that patients with rheumatoid arthritis have asymptomatic 'non-significant' Proteus mirabilis bacteriuria more frequently than healthy controls. J Infect 1999;38:99-106.

8. Tishler M, Caspi D, Almog Y, Segal R, Yaron M. Increased incidence of urinary tract infection in patients with rheumatoid arthritis and secondary Sjögren's syndrome. Ann Rheum Dis 1992;51:604-6.

9. Vitali C, Bombardieri S, Jonsson R, Moutsopoulos HM, Alexander EL, Carsons SE, et al. Classification criteria for Sjögren's syndrome: a revised version of the European criteria proposed by the AmericanEuropean Consensus Group. Ann Rheum Dis 2002;61:554-8.

10. Aletaha D, Neogi T, Silman AJ, Funovits J, Felson DT, Bingham CO 3rd, et al. 2010 Rheumatoid arthritis classification criteria: an American College of Rheumatology/European League Against Rheumatism collaborative initiative. Arthritis Rheum 2010;62:2569-81.

11. Gemalmaz H. BPH'de klinik uygulama kılavuzları. Türk Üroloji Dergisi 2007;33:92-9.

12. Irlapati RV, Nagaprabu VN, Suresh K, Agrawal $\mathrm{S}$, Gumdal N. Infections in rheumatology practice: an experience from NIMS, Hyderabad. Indian $\mathrm{J}$ Rheumatol 2011;6:25-30.

13. Rojas-Serrano J, Cardiel MH. Lupus patients in an emergency unit. Causes of consultation, hospitalization and outcome. A cohort study. Lupus 2000;9:601-6.

14. Noël V, Lortholary O, Casassus P, Cohen P, Généreau $\mathrm{T}$, André $\mathrm{MH}$, et al. Risk factors and prognostic influence of infection in a single cohort of 87 adults with systemic lupus erythematosus. Ann Rheum Dis 2001;60:1141-4.

15. Duffy KN, Duffy CM, Gladman DD. Infection and disease activity in systemic lupus erythematosus: a review of hospitalized patients. J Rheumatol 1991;18:1180-4.

16. Nived O, Sturfelt G, Wollheim F. Systemic lupus erythematosus and infection: a controlled and prospective study including an epidemiological group. Q J Med 1985;55:271-87.

17. Shearn MA, Tu WH. Nephrogenic diabetic insipidus and other defects of renal tubular function in sjoergren's syndrome. Am J Med 1965;39:312-8.

18. Lemp MA, Gold JB. An in vivo study of the corneal surface in keratoconjunctivitis sicca. Trans Ophthalmol Soc U K 1985;104:436-8.

19. Shibata S, Ubara Y, Sawa N, Tagami T, Hosino $J$, Yokota $M$, et al. Severe interstitial cystitis associated with Sjögren's syndrome. Intern Med 2004;43:248-52.

20. Warren JW, Abrutyn E, Hebel JR, Johnson JR, Schaeffer AJ, Stamm WE. Guidelines for antimicrobial treatment of uncomplicated acute bacterial cystitis and acute pyelonephritis in women. Infectious Diseases Society of America (IDSA). Clin Infect Dis 1999;29:745-58.

21. Czaja CA, Scholes D, Hooton TM, Stamm WE. Population-based epidemiologic analysis of acute pyelonephritis. Clin Infect Dis 2007;45:273-80.

22. Livermore DM. beta-Lactamases in laboratory and clinical resistance. Clin Microbiol Rev 1995;8:557-84. 